\title{
论 文
}

\section{新型冲量式谷物联合收割机智能测产系统}

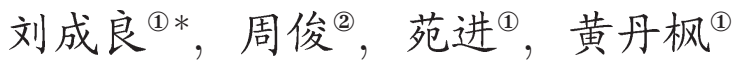 \\ (1) 上海交通大学机电控制研究所, 上海 200030 \\ (2) 南京农业大学工学院, 南京 210031 \\ *通信作者. E-mail: chlliu@sjtu.edu.cn
}

收稿日期: 2010-05-10；接受日期: 2010-08-13

国家高技术研究发展计划 (批准号: 2006AA10A305, 2006AA10A311) 资助项目

\begin{tabular}{l|l|l} 
摘要 作物产量分布信息是精准农业的关键基础数据. 以数字型冲量式谷 & 关键词 \\
物质量流量传感器为核心部件, 应用 CAN 总线架构设计了联合收割机测产 & 谷物质量流量传感器 \\
系统. 设计了双平行梁结构的冲量式谷物质量流量传感器, 其中第一根平行 & 测产 \\
梁上的拦截指直接受谷物籽粒流冲击; 第二根平行梁则只用于检测收割机振 & 精准农业 \\
动以及地形变化引起机器倾斜等干扰, 不与谷物籽粒流接触. 采用最小二乘 & 联合收割机 \\
法求取自适应对消因子, 利用第二根平行梁输出作为参考信号进行自适应干 & \\
扰对消, 有效地消除了第一根测量平行梁中各种干扰引起的输出. 大田初步 & \\
实验结果表明, 最大相对测产误差小于 $4 \%$. &
\end{tabular}

\section{1 引言}

谷物联合收割机测产系统在田间作业过程中, 利用 GPS 定位系统确定收割机的位置, 根据收割机 的速度、割幅和谷物籽粒的流量可测出各小区地块的产量, 绘出产量分布图. 这是下一季变量作业的 决策依据, 同时又可以来评价前一季精准管理的实效, 进而修正相关决策模型, 使精准农业成为了完 整的闭环调节系统.

谷物质量流量传感器是测产系统的核心部件, 其测量精度、抗干扰性能对产量图的准确性有决定 性的意义 [1]. 目前, 联合收获机使用的谷物流量传感器有容积式、辐射式、冲量式等. 其中冲量式流 量传感器由于结构简单、成本低廉而得到广泛的应用 ${ }^{[2 ~ 4]}$; 但是机器的振动、谷物含水率、谷物的种 类、流量变化和田间的坡度等因素对测量精度影响很大 ${ }^{[4 \sim 6]}$, 其中联合收获机工作时的振动以及地形 变化引起的各种倾斜对测产误差影响最为显著.

欧美等国经过多年的研究, 目前已经有商品化的测产系统出售, 但是振动和机身倾斜等因素造成 的测产误差仍然存在. Colvin 等 ${ }^{[7]}$ 提出了一种用倾斜传感器测量联合收获机姿态的方法消除倾斜产 生的误差. Fulton 等 [8] 对 John deer 测产系统进行倾斜和俯仰实验发现测产系统的误差与联合收获 机的倾斜和俯仰角度成线性关系, 用倾角传感器修正后系统产生倾斜误差显著降低. Loghavi 等 ${ }^{[9]}$ 通 过对 Fieldstar 谷物测产系统倾斜特性的研究发现, 倾斜导致谷物流量传感器产生了显著的零点漂移, 在斜坡上重新校准零点可以减少倾斜误差, 但当联合收获机处于水平后需要重新调节零点. 
国内测产系统的研究也较多, 上海交通大学是较早从事测产系统研究的单位之一, 首先提出采用 平行梁结构设计谷物流量传感器, 获得了国家发明专利, 分别从敏感元件结构 [10]、阻尼 [11] 以及传感 器信号处理 [12] 等多角度进行了深入研究, 但田间实际测产的相对误差仍然不理想 ${ }^{[13]}$. 胡均万等在此 基础上, 通过增加一个检振平行梁, 利用双板差分方法来进一步消除联合收割机工作振动给传感器精 度造成的影响 [14], 以及机身倾斜变化引起传感器零点漂移 ${ }^{[15]}$. 但这种模拟差分方法由于检振梁和测 量梁在结构和参数等方面很难完全一致, 精度依然难以保证, 并且当传感器元器件改变或工作条件变 化就需要重新整定调节电路参数, 方法麻烦.

因此, 本文首先将在数字传感器基础上设计联合收割机测产系统, 然后阐明使用自适应干扰对消 的方法来进一步提高测产系统精度.

\section{2 智能测产系统组成}

联合收割机测产系统在收割作业过程中测量各小区的产量信息:

$$
Y=\frac{M(1-k)}{W V} .
$$

式中, $M$ 为谷物质量流量, $W$ 为割幅宽度, $V$ 为收割速度, $k$ 为含水率. 这些参数都可以分别用相应的 传感器测量出来后, 送到中心控制计算机处理后, 得到产量数据, 以规定格式存储, 并绘制产量分布图.

为了避免模拟信号的传输干扰, 便于系统扩展, 所有传感器都设计全数字型. 采用 CAN 总线众多 传感器连接在一起 (图 1), CAN 总线是理想的测控系统通信网络, 其数据通信可靠性高、实时性强、 灵活性好, 不但能实现多主方式工作, 而且能在苛刻环境中保证测控装置安全、可靠地完成测量与控 制任务 ${ }^{[13]}$, 非常适合联合收割机这一应用对象. 根据 CAN 协议的仲裁机制, 报文识别符越小的节点 具有越高的优先权, 当总线发生冲突时, 优先级高的先发送, 其他节点自动退出总线. 工作中, 中心控 制计算机首先查询各传感器的连接状态, 各传感器节点收到查询信号后将反馈白身的信息. 中心计算 机根据收到的信息判断并显示测产系统的状况, 设置相应的系统参数默认值. 查询应答后, 个传感器 节点将定时主动把测量信息发送给中心计算机.

\section{3 双平行梁谷物质量流量传感器}

\section{1 传感器结构}

谷物质量传感器是测产系统的核心组件, 它一般安装在联合收获机净粮升运器顶部, 当谷物在升 运器作用下不断地冲击到传感器拦截指上时, 则会引起平行梁弹性元件横向变形, 此变形再经粘固其 上的电阻应变片电桥转换成电量信号, 此信号与谷物质量流量在一定范围内呈近似线性关系. 但是, 同 时也受到谷物颗粒的物理特性、安装姿态以及实际流量等多种因素的影响.

由于是通过测冲击力来检测谷物质量流量, 所以冲量式传感器本质上易受安装基础振动以及安装 角度等因素的干扰. 并且, 联合收割机本身又恰好是移动机械, 无论是地面激励还是自身工作部件, 不 平衡振动都比较大. 在前续研究中, 我们发明了双孔平行梁传感器, 并从敏感元件结构、阻尼以及输出 信号处理等多角度来抑制这些干扰, 但效果仍然不理想. 因此, 这里在单双孔平行梁谷物质量流量传 感器基础上再添加一个检振平行梁, 用来测量收割机干扰振动以及倾斜等情况, 以便于利用自适应干 扰对消办法进一步滤除这些干扰. 


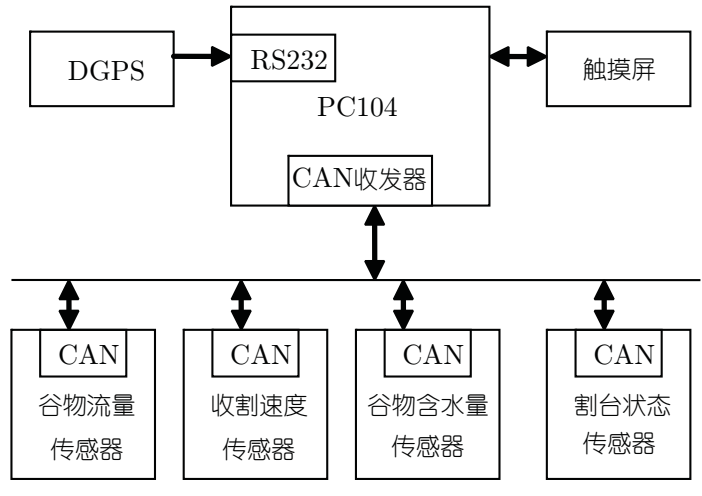

图 1 智能测产系统结构图

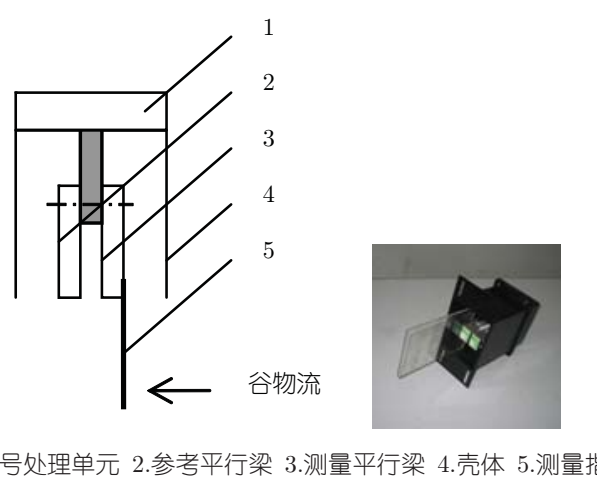

图 2 双平行梁谷物质量流量传感器结构示意图

如图 2 所示, 双平行梁谷物质量流量传感器具有 2 根平行梁, 即 1 根直接接受谷物籽粒流冲击的 测量平行梁, 以及用于敏感收割机振动或倾斜的参考平行梁. 2 根平行梁输出在信号处理单元完成信 号数字化和干扰对消.

其中对于单个双孔平行梁弹性元件而言, 由于其对称的结构, 当与适宜的电桥电路配合后, 就可 以实现只对被测正向力敏感, 而对空间其他 5 个力/力矩不输出, 同时又能够保证不受被测力作用点 变化的影响. 这样就可以只对谷物质量流产生的正向冲击力敏感, 而又不受其冲击作用点的影响. 图 3 是应变片电桥在双孔平行梁上粘贴的示意图, 图 4 是对应的应变片电桥电路, 设计中使用了金属箔式 电阻应变片, 阻值为 $350 \Omega$.

联合收割机田间工作时谷物颗粒流在升运器的带动下冲击在传感器拦截指上, 进而引起平行梁弹 性元件变形. 但是, 冲击力 $P$ 的作用点会随传感器安装位置、谷物瞬时质量流量、升运器工作速度等 因素的变化而改变, 作用在平行梁上的等效力矩 $M$ 就成为一变量, 因此, 传感器正确工作的前提应该 是其输出不受力矩 $M$ 的影响, 只与冲击力 $P$ 成比例. 图 4 中的 $U$ 是供桥电压, $U_{i}$ 为电桥输出信号. $R_{3}$ 两端并联了一个偏置电阻 $R_{x}$, 是为了调节电桥输出. 根据平行梁变形与电阻值之间的变化关系, 可 以得到

$$
U_{i}=-\frac{K U}{4 E W} P L+\frac{R}{4 R_{x}} U
$$

式中, $E$ 为梁材料的弹性模量, $W$ 为各应变片处平行梁的抗弯截面模量, $R$ 为应变片未变形时的电 阻值.

由公式 (2) 明显可见, 电桥输出与冲击作用力 $P$ 成比例, 而与平行梁上作用的等效力矩没有任何 关系. 偏置电阻的增加没有改变传感器线性度, 只是增加了一个固定的偏置电压.

\section{2 传感器自适应干扰对消}

低频谷物籽粒流在谷物升运器的带动下作用在传感器上, 其产生的信号与联合收割机中众多不平 衡工作部件以及地面引起的振动干扰频带有重叠, 并且此振动干扰频率值会随着联合收割机的工况变 化、地形改变而在较大范围内随机漂移. 使用简单的滤波方法, 不但会损害有用信号, 而且也无法适应 联合收割机的工况改变. 


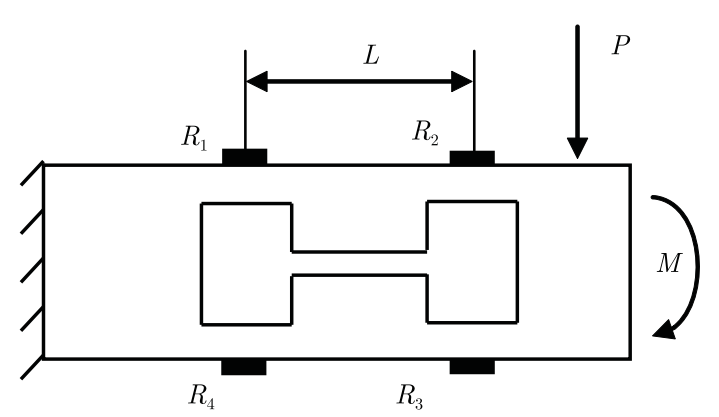

图 3 应变片电桥粘贴位置示意图

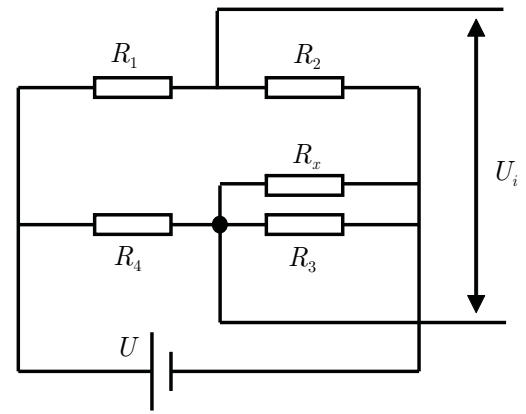

图 4 应变片电桥电路

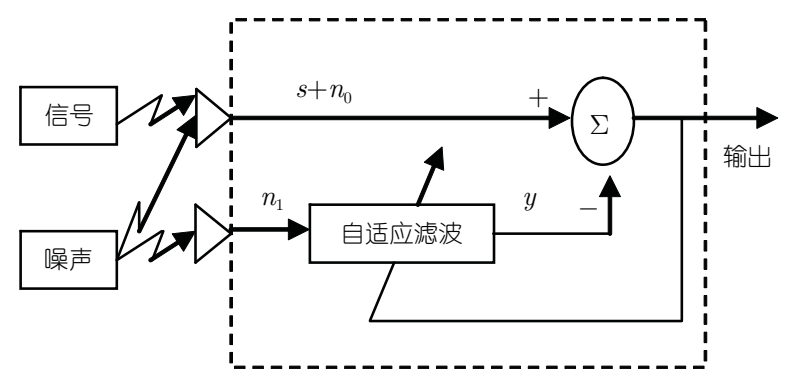

图 5 自适应干扰对消

为了消除这种干扰噪声, 采用图 5 所示的自适应干扰对消方法. 它有检振平行梁和测量平行梁 2 个传感器输入, 其中测量平行梁除接收到谷物籽粒冲击信号 $s$ 外, 还收到 1 个与信号不相关的振动噪 声 $n_{0}$, 即总输入为 $s+n_{0}$. 由于 2 个传感器参数存在差异, 所以检振平行梁必然将接收到与产量信号 不相关的而以某种其他方式与振动噪声 $n_{0}$ 相关的噪声 $n_{1}$. 自适应干扰对消器就是将振动噪声 $n_{1}$ 加 以滤波, 使白适应滤波器产生出与噪声 $n_{0}$ 相匹配的输出信号 $y$. 然后将该输出从原始输入中减去, 最 后产生系统输出

$$
\varepsilon=s_{0}+n_{0}-y .
$$

$\varepsilon$ 将用来控制白适应滤波器, 以使系统输出逐渐逼近信号源 $s$.

工作中, 首先对 2 个平行梁的输出信号按同一采样频率进行采样量化. 在某一时刻取测量平行梁 最近的 $N$ 个采样值 $x(n)(n=0,1,2, \ldots, N-1)$, 稍后再取检振平行梁输出信号的最近 $Q(Q>N)$ 个 采样值 $z(n)(n=0,1,2, \ldots, Q-1)$, 目前实验中 $N$ 取值为 $200, Q$ 等于 $1.5 N$. 从 $z(n)$ 的起始点逐点 进行相关运算, 得到相关序列:

$$
r(p)=\sum_{n=0}^{N-1} x(n) z_{p}(n)(p=0,1,2, \ldots, Q-N) .
$$

式中, $z_{p}(n)$ 是 $z(n)$ 中从起始点处依次取长度为 $N$ 的子序列.

这样, 计算最大相关系数 $r(m)$ 为

$$
r(m)=\max [r(p)]
$$

式中, $m$ 即为 $z(n)$ 中与 $x(n)$ 具有最大相关系数的子序列 $z_{m}(n)$ 的起点. 


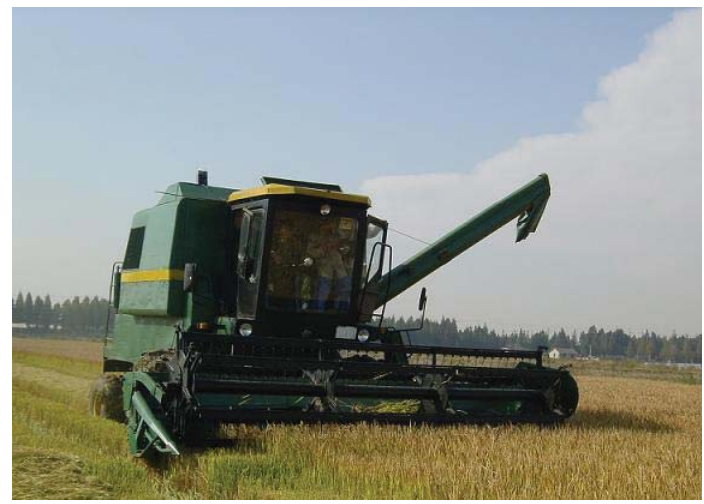

图 6 大田试验

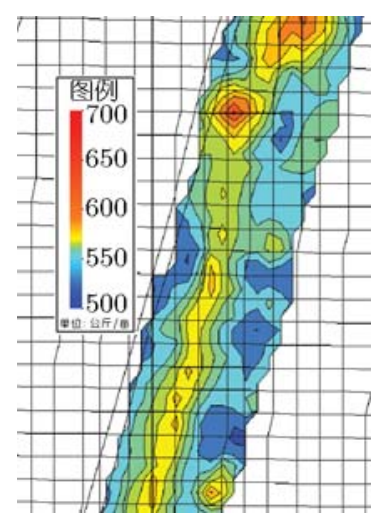

图 7 产量分布图

$z_{m}(n)$ 保证了与 $x(n)$ 的波形的最大统计相似性, 但不保证有最小能量误差. 为了最大程度地对消 振动干扰, 采用最小二乘法, 求取对消因子 $w$. 设对消后误差信号为

$$
\varepsilon(n)=x(n)-w z_{m}(n) .
$$

则误差能量为

$$
\sum_{n=0}^{N-1} \varepsilon^{2}(n)=\sum_{n=0}^{N-1}\left[x(n)-w z_{m}(n)\right]^{2} .
$$

根据最小二乘法, 公式 (7) 对 $w$ 求一阶导数并令之等于 0 , 可得最小能量误差的条件为

$$
w=\frac{\sum_{n=0}^{N-1} x(n) z_{m}(n)}{\sum_{n=0}^{N-1}\left[z_{m}(n)\right]^{2}} .
$$

得到对消因子后, 带入公式 (6), 就可实时地计算出干扰对消后的传感器信号. 这种干扰无论是收 割机工作振动干扰, 还是地形变化引起的零点漂移, 都可被有效滤除. 由于对消因子是在线计算的, 因 此, 并不需要在测产系统工作前对传感器进行麻烦的调节准备, 同时也可更好地适应收割机不断改变 的工况.

\section{4 大田实验}

在上海五四农场开展了田间测产实验, 作物对象是水稻, 收割机型号是 JL1075 (图 6). 谷物质量 流量传感器安装在收割机净粮升运器的出口处, 采用亚米级差分 GPS 实时记录收割机位置, 通过测量 车轮转速来近似获取联合收割机作业速度. 收割过程中, 测产系统每隔约 $4.86 \mathrm{~m}$ 等间距计算小区平均 产量. 采用文献 [13] 中相同的方法, 由于田间小范围区域内作物单位面积产量近似恒定, 利用离线称 重法测量作物平均产量, 然后以之为基准计算测产系统的相对误差, 结果在 $4 \%$ 范围内. 最后, 采用距 离反比加权插值法, 绘制的水稻产量图如图 7 所示.

\section{5 结论}

基于 CAN 总线框架, 设计了联合收割机智能测产系统. 使用双平行梁结构设计了冲量式谷物质 量流量传感器, 采用最小二乘法在线计算自适应对消因子, 有效地进行了干扰对消, 滤除了收割机工 
作振动给传感器造成的干扰以及地形变化引起的传感器零点漂移. 大田测产实验结果表明, 相对误差 小于 $4 \%$.

\title{
参考文献
}

1 Grisso R D, Jasa P J, Schroeder M A, et al. Yield monitor accuracy successful farming magazine case study. Appl Eng Agric, 2002, 18: 147-151

2 Reyns P, Missotten B, Ramon H, et al. A review of combine sensors for precision farming. Precis Agric, 2002: 169-182

3 Lee C K, Iida M, Kaho T, et al. Development of impact type sensor for heading feeding combine. J JSAM, 2000, 62: $81-88$

4 Koichi S, Tsuneo K, Hisashi H. Impact-based grain yield sensor with compensation for vibration and drift. J JSAM, 2002, 64: 108-115

5 Grisso R D, Jasa P J, Schroeder M A. Yield monitor accuracy: successful farming magazine case study. Appl Eng Agric, 2002, 18: 147-151

6 Burks T F, Shearer S A, Fulton J P. Effects of time-varying inflow rates on combine yield monitor accuracy. Appl Eng Agric, 2004, 20: 269-275

7 Colvin T S, Arslan S. A review on yield reconstruction and sources of errors in yield maps. In: Proceedings of the 5th International Conference on Precision Agriculture. Bloomington Minnesota, 2000. 33-39

8 Fulton J P, Sobolik C J, Shearer S A, et al. Grain yield monitor flow sensor accuracy for simulated varying field slopes. Appl Eng Agric, 2009, 25: 15-21

9 Loghavi M, Ehsani R, Reeder R. Development of a portable grain mass flow sensor test rig. Comput Electron Agric, 2008, 61: 160-168

10 周俊, 刘成良. 平行梁冲量式谷物质量流量传感器弹性元件设计. 农业工程学报, 2007, 23: 110-114

11 周俊, 周国祥, 苗玉彬, 等. 悬臂梁冲量式谷物质量流量传感器阻尼设计. 农业机械学报, 2005, 36: 121-123

12 周俊, 刘成良. 平行梁冲击式谷物质量流量传感器信号处理方法. 农业工程学报, 2008, 24: 183-187.

13 周俊, 苗玉涁, 张风传, 等. 平行梁冲量式谷物质量流量传感器田间实验. 农业机械学报, 2006, 37: 102-105

14 胡均万, 罗锡文, 阮欢, 等. 双板差分冲量式谷物流量传感器设计. 农业机械学报, 2009, 40: 69-72

15 胡均万, 罗锡文, 陈树人, 等. 机身倾斜导致谷物流量传感器零点漂移的补偿. 农业机械学报, 2009, 40(增刊): 57-60

\section{Yield monitor system based on impact-based grain mass sensor}

\author{
LIU ChengLiang ${ }^{1}$, ZHOU Jun ${ }^{2}$, YUAN Jin ${ }^{1} \&$ HUANG DanFeng ${ }^{1}$ \\ ${ }^{1}$ Mechatronics Institute, Shanghai Jiaotong University, Shanghai 200030, China; \\ ${ }^{2}$ College of Engineering, Nanjing Agricultural University, Nanjing 210031, China
}

Abstract Yield map is the key basic data for precision agriculture. With digital impact-based grain mass sensor as the core component, a yield monitor system was designed based on the framework of CAN bus. The new impact-based grain mass sensor was designed by using two parallel beam load cells, and the first one was impacted by grain flow directly, the second one was used for measuring the sensor base vibration caused by the combine harvester or the combine inclination caused by the uneven terrain. The least square method was applied to calculate the adaptive cancellation factor on line. And the adaptive disturbance cancellation was carried out with the output signal of the second parallel beam as the reference. The field experiment results showed that the relative error was within $4 \%$

Keywords impact-based grain mass sensor, yield monitor, precision agriculture, combine 